\title{
MRI and CT in the diagnosis of coronary artery disease: indications and applications
}

\author{
Konstantin Nikolaou • Hatem Alkadhi • \\ Fabian Bamberg • Sebastian Leschka • \\ Bernd J. Wintersperger
}

Received: 30 August 2010 /Revised: 27 September 2010 / Accepted: 19 October 2010 /Published online: 23 November 2010

(C) European Society of Radiology 2010

\begin{abstract}
In recent years, technical advances and improvements in cardiac computed tomography (CT) and cardiac magnetic resonance imaging (MRI) have provoked increasing interest in the potential clinical role of these techniques in the non-invasive work-up of patients with suspected coronary artery disease (CAD) and correct patient selection for these emerging imaging techniques. In the primary detection or exclusion of significant CAD, e.g. in the patient with unspecific thoracic complaints, and also in patients with known CAD or advanced stages of CAD, both $\mathrm{CT}$ and MRI yield specific advantages. In this review, the major aspects of non-invasive MR and CT imaging in the diagnosis of CAD will be discussed. The first part describes the clinical value of contrast-enhanced non-invasive $\mathrm{CT}$ coronary angiography (CTCA), including the diagnostic accuracy of CTCA for the exclusion or detection of significant $\mathrm{CAD}$ with coronary artery stenoses that may
\end{abstract}

K. Nikolaou $(\bowtie) \cdot$ F. Bamberg $\cdot$ B. J. Wintersperger

Department of Clinical Radiology, University Hospitals Munich, Grosshadern Campus, Marchioninistr. 15,

81377, Munich, Germany

e-mail: konstantin.nikolaou@med.lmu.de

H. Alkadhi

Institute of Diagnostic and Interventional Radiology,

University Hospital,

Zurich, Switzerland

S. Leschka

Institute of Radiology, General Hospital,

Saint Gall, Switzerland

\section{B. J. Wintersperger}

University Health Network,

Mount Sinai Hospital and Women's College Hospital,

University of Toronto,

Toronto, Canada require angioplastic intervention, as well as potentially valuable information on the coronary artery vessel wall. In the second section, the potential of CT for the imaging of myocardial viability and perfusion will be highlighted. In the third and final part, the range of applications of cardiac MRI in CAD patients will be outlined.

Keywords Coronary artery disease $\cdot$ Diagnosis $\cdot$ CT $\cdot$ MRI

\section{Introduction}

In recent years, technical advances and improvements in cardiac computed tomography (CT) and cardiac magnetic resonance imaging (MRI) have provoked increasing interest in the potential clinical role of these techniques in the noninvasive work-up of patients with suspected coronary artery disease (CAD) and correct patient selection for these emerging imaging techniques. In the primary detection or exclusion of significant CAD, e.g. in the patient with unspecific thoracic complaints, and also in patients with known CAD or advanced stages of CAD, both CT and MRI yield specific advantages.

Most established non-invasive diagnostic techniques in the primary diagnostic work-up of patients with suspected CAD, such as stress electrocardiography (ECG), stress echocardiography or scintigraphy [single photon emission computed tomography (SPECT) and positron emission tomography (PET)] are aimed at the detection of a reduced coronary perfusion reserve, i.e. detection of reduced myocardial perfusion or regional wall motion abnormalities under stress conditions. In recent years, MRI and CT have also increasingly been used in the primary diagnostic workup of CAD. Such primary diagnosis of CAD by MRI and/ or CT can utilise different strategies: detection of coronary 
calcifications; imaging coronary artery stenoses; and detection of a reduced coronary perfusion reserve (resulting in restricted myocardial perfusion with or without impairment of myocardial function). For these indications, CT and MRI applications available are:

1. Coronary calcification scoring (or coronary vessel wall imaging in general) by $\mathrm{CT}$

2. Non-invasive coronary angiography by MRI or CT

3. Stress cine MRI and stress perfusion MRI

4. Myocardial viability imaging using MRI or CT

Recently, the first results of stress perfusion CT have also been published. Many of these imaging methods have been reported to yield a high negative predictive value, i.e. a normal result of these tests rules out a haemodynamically significant $\mathrm{CAD}$ with a high probability. For a reasonable implication of imaging strategies in clinical practice, however, the pre-test probability must be considered to avoid needless examinations [1]. High pre-test probability invariably demands invasive coronary angiography for planning or performing revascularisation therapy. Intermediate pre-test probability, on the contrary, justifies deferring further imaging studies, if MRI and/or CT are/is normal.

Cardiac MRI has matured into a multipurpose noninvasive imaging tool for the assessment of ischaemic cardiomyopathy. The breadth of applications possible with cardiac MRI allows combined non-invasive assessment of myocardial perfusion, function and myocardial viability - a task that usually requires use of myocardial scintigraphy and echocardiography. As such, cardiac MRI currently holds a strong position in the non-invasive work-up of patients with CAD. The distinct advantages of MRI over current conventional nuclear-based cardiac-imaging techniques, such as PET or myocardial scintigraphy, include its high spatial resolution and lack of exposure of the patient to ionising radiation. Also, quantification of cardiac morphology and function by MRI is more accurate and image quality is more reproducible than in echocardiography, independent of the operator's experience and skill level or the patient's anatomy.

On the other hand, the technical parameters of cardiac $\mathrm{CT}$, such as the temporal and spatial resolution, have shown a dramatic improvement over the last few years. Today's system generations reach a spatial resolution of up to $0.5 \mathrm{~mm}$, with a temporal resolution of less than $50 \mathrm{~ms}$ and imaging times of less than $1 \mathrm{~s}$, depending on the CT systems used. With an increasing number of cases permitting excellent visualisation of both coronary artery lumen and wall, and based on many publications suggesting high sensitivities and specificities for the detection or exclusion of significant coronary stenosis, CT coronary angiography (CTCA) is being adopted into clinical practice more and more frequently. However, imaging of myocardial viability and quantification of myocardial perfusion with CT has not yet become a clinical reality, even if these applications would greatly complement non-invasive coronary CT angiography.

The review and outlook presented here will focus on three major aspects of non-invasive MR and CT imaging in the diagnosis of CAD. The first part describes the clinical value of contrast-enhanced non-invasive $\mathrm{CT}$ coronary angiography, including the diagnostic accuracy of CT coronary angiography for the exclusion or detection of significant CAD with coronary artery stenoses that may require angioplastic intervention, as well as potentially valuable information on the coronary artery vessel wall. In the second section, the potential of CT for imaging of myocardial viability and perfusion will be highlighted. In the third and final part, the range of applications of cardiac MRI in CAD patients will be outlined. Not all the imaging methods described in this review are already being used in the daily clinical work-up of patients with CAD, but they are being used more and more every day. Therefore, their correct clinical indication and clinical value need to be discussed.

\section{CT coronary angiography: luminography and beyond}

CT coronary angiography: are we there yet?

Coronary catheter angiography (CCA) is still considered to be the clinical standard technique for the evaluation of CAD. However, the risk of serious complications associated with CCA is not negligible, and inconvenience for the patient and economic deliberations have strengthened the search for a non-invasive alternative. Currently, CT is the most promising imaging technique for the non-invasive evaluation of the coronary artery tree. Current CT technology combines high spatial and temporal resolution with fast volume coverage, thus enabling imaging of the small and fast-moving coronary arteries within a single breath-hold.

A huge number of publications have abounded evaluating the diagnostic performance of CT coronary angiography. With continuous development in CT system generations-starting with four-slice CT until the advent of the most recent 320-slice and second-generation dualsource CT systems - the diagnostic accuracy performs as follows [2]. Patients with significant morphological coronary stenoses are detected with a sensitivity of $95 \%$ or higher. With modern technology, the specificity to detect patients with significant morphological coronary artery stenoses with CT showed steady improvement. In parallel, the number of segments showing a non-diagnostic image quality decreased. Most importantly, the negative predictive value of CT coronary angiography has been reported in 
many studies to be as high as $99-100 \%$, indicating the excellent ability of this non-invasive imaging technique to rule out significant morphological CAD (Fig. 1).

The most important clinical indication for CT coronary angiography is the evaluation of chest pain syndrome in patients with intermediate pre-test probability of CAD and uninterpretable ECG, or the inability of the patient to undergo exercise testing [3]. On the other hand, patients with a high pre-test probability of CAD should not undergo CT coronary angiography. Similarly, in patients with a low pre-test probability, the appropriateness of CT coronary angiography is considered uncertain. In a clinical context, CT should be used as a filter test: When the CT coronary angiography test results are negative, no further invasive work-up is required, and when the results are positive, CCA should subsequently be performed in most cases.

Despite these performance characteristics, CT is associated with a number of shortcomings. First, elevated and irregular heart rates still pose a challenge for CT coronary angiography. Although this challenge has been partly overcome with most recent $\mathrm{CT}$ systems having a very high temporal resolution of up to $43 \mathrm{~ms}$, a decrease in image quality is still present in patients with combined high and irregular heart rates. Another limitation is represented by severe calcifications in the arterial vessel walls, causing socalled blooming artefacts. This hinders the evaluation of the coronary artery lumen, eventually causing an overestimation of the degree of stenoses (and thus, a false-positive CT result). Finally, an important draw-back of CT is the radiation exposure associated with the technique [4]. With earlier CT generations, effective radiation doses of $>20 \mathrm{mSv}$ have been reported. In the meantime, however, various radiation dose-saving algorithms have been developed [5] that all prove efficient in the reduction of the radiation exposure, to a dose that is similar or below that reported for CCA. Two of these radiation dose-saving techniques should be mentioned briefly:

First, prospectively ECG-gated CT coronary angiography, also called the "step-and-shoot" mode, has been introduced. This mode is characterised by turning on the $\mathrm{X}$-ray tube only at a predefined time point of the cardiac cycle, usually in mid-diastole, while keeping the patient table stationary. The X-ray exposure time of this technique is short, and thus, low radiation doses, ranging between 1.2 and $4.3 \mathrm{mSv}$, have been reported using various 64-slice and first-generation dual-source 64-slice CT systems [6-8]. Most importantly, this low-dose CT coronary angiography technique was still accurate in the diagnosis of coronary artery stenoses [9].

Second, along with the introduction of the recent secondgeneration dual-source CT, the high-pitch mode has been introduced for cardiac CT [10-13]. In this mode, data acquisition is also prospectively triggered with the ECG of the patient. However, the data are acquired in a spiral mode, with the CT table running with a very high pitch of 3.4. Using this high-pitch mode, the entire heart can undergo imaging within only one single diastole. Various reports have indicated that this technique delivers not only a lowradiation dose of around or below $1 \mathrm{mSv}$ for a cardiac study, but also showed that this mode provides diagnostic image quality [10-13]. Similar to the step-and-shoot mode, the high-pitch mode is also characterised by high diagnostic performance [14].
Fig. 1 CT coronary angiography in a 55-year-old male patient with atypical chest pain demonstrating normal coronary arteries. a Right coronary artery (RCA); b left anterior descending coronary artery (LAD), c left circumflex coronary artery (LCX), with no plaques or stenoses. In this patient, CT reliably ruled-out $\mathrm{CAD}$; no subsequent invasive catheter coronary angiography is required
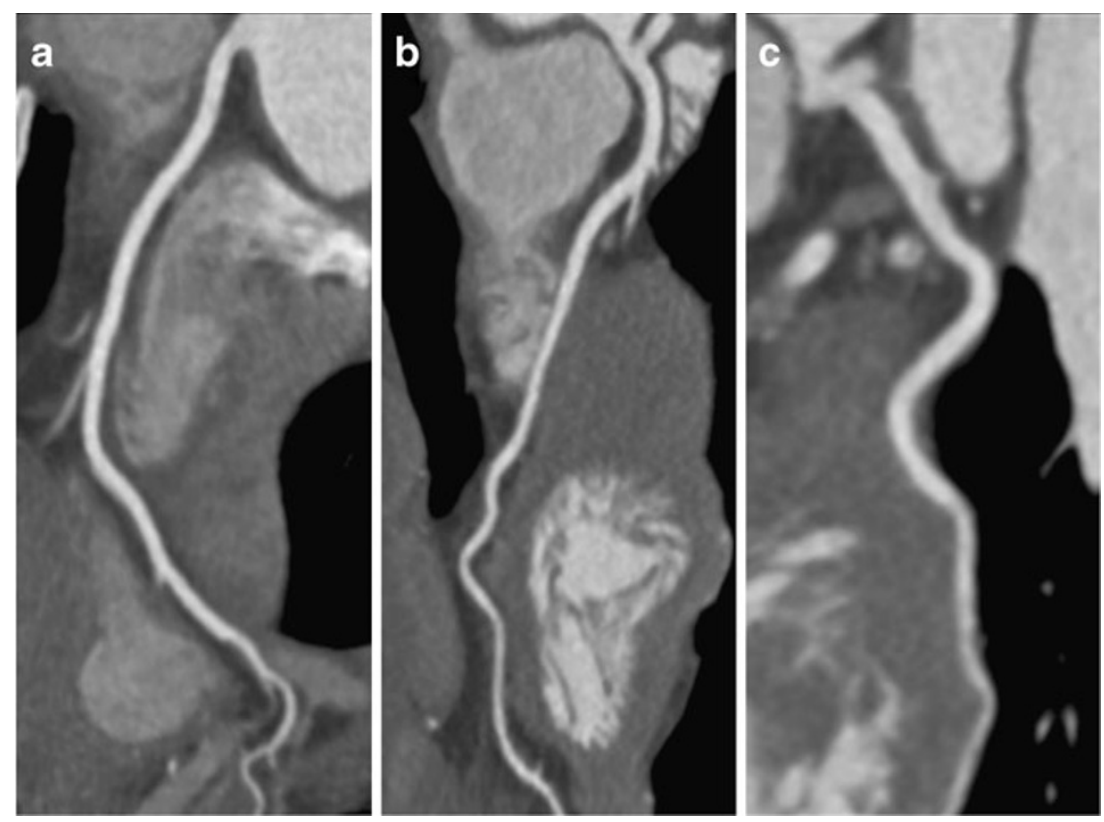


\section{CT for coronary plaque imaging}

The imaging reference standard for the detection and characterisation of coronary artery plaques in patients is intravascular ultrasound (IVUS). Owing to its excellent spatial and contrast resolution, IVUS is able to characterise and classify coronary artery plaques with a high accuracy compared with histopathology. However, IVUS is an invasive technique and thus not feasible in asymptomatic patients, i.e. in a preclinical setting. Thus, a non-invasive imaging test with the ability to characterise and quantify features of an individual coronary artery plaque and the overall coronary atherosclerotic plaque burden could have a tremendous effect on the prevention and treatment of CAD [15].

The advantage of CT coronary angiography-beyond its ability to image the degree of luminal stenosis - is the simultaneous detection and characterisation of coronary atherosclerotic plaques. In contrast-enhanced CT coronary angiography, imaging of calcified, mixed and non-calcified coronary plaques is possible (Fig. 2). Thus, research targeting the qualitative and quantitative assessment of coronary plaques, including the assessment of plaque size, plaque composition and plaque remodelling, has become feasible. Importantly, the relevance of plaque detection and quantification by $\mathrm{CT}$ derives from its independent prognostic value in patients with known or suspected CAD [16, 17]. It is important to note that the normal coronary wall cannot be assessed by $\mathrm{CT}$ because of inherent limitations in spatial resolution. However, plaques of potential clinical importance tend to be larger, having a sizeable necrotic core, and are most often found in the proximal coronary arterial segments. This makes them accessible for visualisation with CT [15].

With 64-slice CT, a sensitivity of $83 \%$ was found for detecting non-calcified coronary artery plaques. Regarding calcified plaques, a higher sensitivity of $95 \%$ was demonstrated [18]. Comparable results were reported by other groups employing 64-slice CT [15], indicating a still suboptimal spatial and temporal as well as contrast resolution of CT. Importantly, observer experience also has a significant impact on the accuracy of plaque detection [19].

Regarding the quantification of coronary arterial plaque volume with CT, correlations are only moderate compared with IVUS. In general, the accuracy varies with coronary arterial plaque composition: volumes of non-calcified and often also mixed plaques are frequently underestimated, whereas the volume of calcified plaque is often overestimated in CT [20]. The latter can be explained by blooming artefacts and by partial volume effects caused by the contrast-enhanced vessel lumen. Finally, standardised and software-based automated assessment of coronary plaques with CT is still lacking [21]. Quantitative coronary artery plaque composition has been repetitively assessed with various $\mathrm{CT}$ generations [22, 23]. Most investigators measured CT numbers (in Hounsfield units) to determine the ability of CT to characterise and to distinguish the various plaque types. However, although early study results were promising, studies have consistently shown that CT numbers of non-calcified plaque components vary widely. Although mean values were often significantly different between fibrous and lipid-rich coronary arterial plaques, a substantial overlap of CT numbers was found, precluding a reliable characterisation and differentiation of the various
Fig. 2 CTCA demonstrating a non-calcified (a), a mixed (b), and a calcified (c) coronary arterial plaque
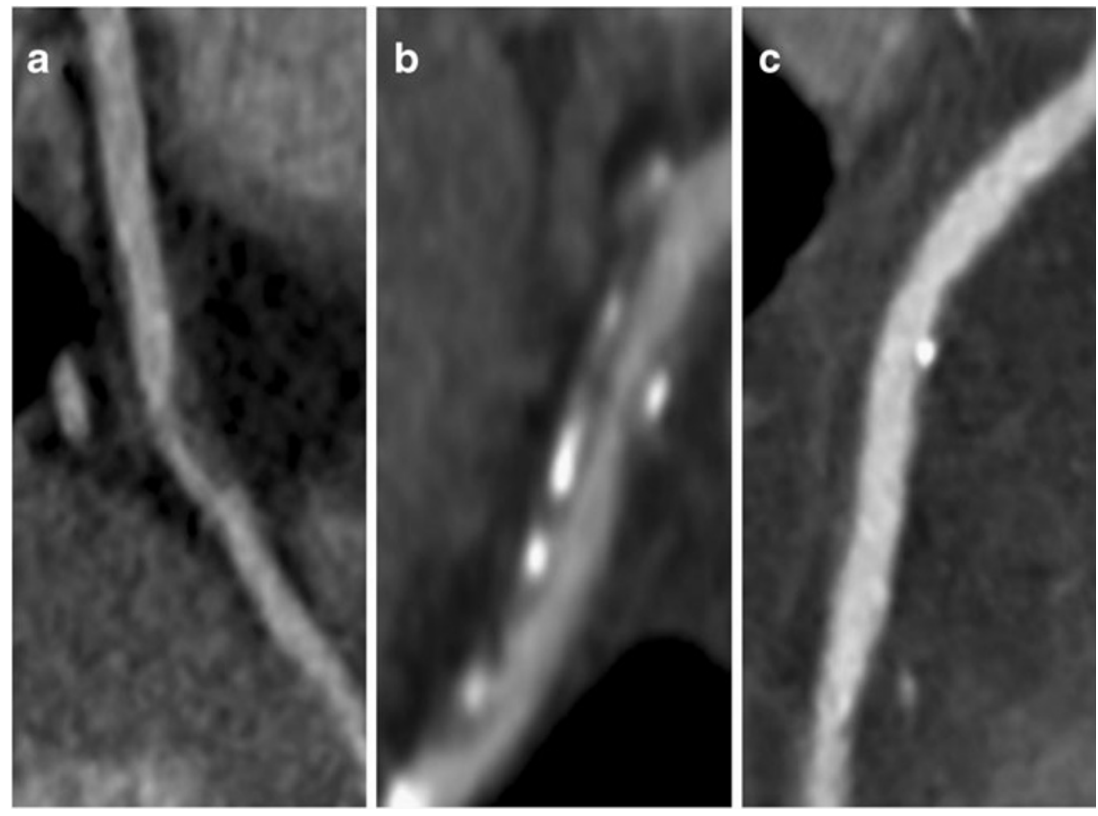
non-calcified coronary arterial plaques [15]. Furthermore, measurements of $\mathrm{CT}$ numbers in coronary plaques should be made with caution, because contrast enhancement within the vessel lumen considerably affects attenuation measurements of the plaques in the adjacent vessel wall [24]. Also, the use of different tissue reconstruction filters affects the CT numbers of coronary arterial plaques [25].

Future directions of $\mathrm{CT}$ for plaque detection and characterisation include a more detailed morphological analysis of coronary plaque types [26], and eventually the use of molecular imaging in adjunct with contrast agents targeting specific cells that are important in the development and evolution of coronary arterial plaques [27].

\section{CT viability und perfusion}

Assessment of myocardial viability and myocardial perfusion plays a critical role in the evaluation of patients with CAD as patterns of myocardial perfusion are associated with short- and long-term prognosis and the haemodynamic relevance of a coronary artery stenosis [28, 29]. Cardiac $\mathrm{CT}$, which has evolved as a useful tool for the assessment of obstructive and non-obstructive plaque, has mainly been limited to the morphological assessment of CAD, i.e. CT coronary angiography [30]. However, imaging of myocardial viability and quantification of myocardial perfusion would greatly complement non-invasive coronary CT angiography. Within recent years, a number of studies have focused on the demonstration of myocardial "perfusion" deficits using multidetector (MD) CT. However, most of the studies used either a single volume data acquisition during the angiographic phase or double exposure with the acquisition of an additional delayed phase data set. However, this approach is based on static imaging rather than on dynamic imaging. Therefore, these kinds of datasets represent more a kind of blood volume distribution (early phase) and a delayed contrast agent distribution, i.e. an approach similar to delayed imaging in MRI [31, 32]. This delayed imaging focuses on the visualisation of myocardial viability, a technique that is commonly used in MRI and has already been reported on in the early days of CT imaging $[33,34]$. "Real" myocardial perfusion imaging with CT techniques would be based on the dynamic visualisation of a contrast agent during its first pass through the cardiac chambers, coronary arteries and myocardium. Within the last decade, the approach of dynamic, i.e. timeresolved, first-pass perfusion imaging has already been extensively evaluated using dynamic contrast-enhanced MRI. It has been shown that the combination of rest and stress studies even allows the calculation of the myocardial perfusion reserve and allows reliable assessment of haemodynamic significant vessel changes [35]. However, while in
MRI the linearity of the contrast agent concentration and the signal intensity is limited to a certain range of concentrations of the contrast agent and varies with sequence parameter changes, CT techniques would offer a major benefit addressing this issue: they provide constant linearity of contrast concentration and density.

\section{CT viability imaging}

Acute ischaemic changes in the myocardium after coronary arterial occlusion consist of disruption of cell membrane function and integrity and increased permeability of small vessel walls. In contrast-enhanced CT coronary angiography, the area of low attenuation within the myocardium primarily reflects myocardial oedema, i.e. a pronounced water content of the myocardium, followed by infiltration of inflammatory cells. Subsequently, over days and weeks, necrotic myocardium is replaced by fibrous and/or fatty tissue, which is also characterised by a reduction of attenuation in $\mathrm{CT}$ in a CTCA, compared with normal myocardium $[36,37]$. A number of initial reports on the application of $\mathrm{CT}$ in patients with myocardial infarction have been promising, including early animal studies [38-40] and experiences with electron beam (EB) CT [41-43]. With the introduction of multislice $\mathrm{CT}$ systems, myocardial viability imaging using CT has experienced renewed interest, with several early case reports showing the capability of the technique to detect myocardial infarctions [44, 45]. In general, one has to distinguish two major acquisition techniques that have been reported using CT for the depiction of myocardial viability: (1) the "typical" CTCA, i.e. an arterial first-pass image of the myocardium, without requiring any additional contrast agent or radiation dose; (2) a dedicated CT late enhancement technique, which would typically be added to a CT coronary angiogram as a second imaging procedure, and thus, requiring additional radiation dose (and typically more contrast agent than CTCA alone).

With the first protocol, i.e. in the arterial first-pass phase after contrast agent injection, a myocardial infarct area is typically detectable as a perfusion defect, i.e. hypodensity of the myocardium, as explained above (Figs. 3 and 4). Such a myocardial hypodensity in the first-pass arterial phase image, however, is not specific to infarctions, as similar findings can occur in cases with severe local ischaemia (but no infarction) or other cardiac diseases causing perfusion heterogeneities, such as hypertrophic cardiomyopathy. Also, myocardial contrast enhancement depends on a number of independent variables, e.g. the contrast agent injection protocol or the cardiac output. That is why a significant variance of measured CT attenuation [Hounsfield units (HU)] can be observed in normal and infarcted myocardium in the arterial first pass, indicating that no absolute $\mathrm{HU}$ values can be defined, but the relative 


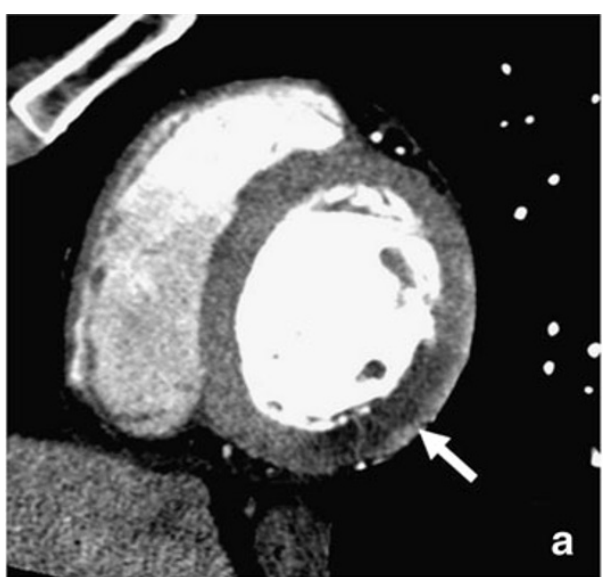

Fig. 3 A 61-year-old male patient with acute myocardial ischaemia and corresponding myocardial perfusion defect from a $85 \%$ coronary stenosis in the left circumflex coronary artery (LCX). Imaging on dual-source CT was performed after percutaneous coronary stent placement in the $\operatorname{LCX}(\mathbf{c})$. a Multiplanar reformat (narrow window) two-chamber view demonstrating the contrast agent-filled left and right ventricle and the hypoperfused lateral-inferior wall (arrow) while the anterior and septal myocardium is homogeneously enhanced. b Longitudinal view of the left ventricle with the perfusion defect of the lateral wall consistent with the LCX lesion measurement of normal and infarcted tissue is the decisive factor [37]. Wall thinning of the left ventricular myocardium is one of the indirect findings associated with the healing process after myocardial infarction, caused by scarring. When it comes to the exact sizing of myocardial infarctions during the arterial first pass of the contrast agent, several studies have reported that CT underestimates the true extent of a myocardial infarction [37, 41]. This might be related to patchy and subendocardial infarctions, as collateral perfusion in the area of infarcted myocardium can obscure foci of necrosis surrounded by normal myocardium. In conclusion, one should interpret infarct size as assessed by contrast-enhanced, arterial phase MDCT as an approximation to the true infarct size rather than as an absolute measurement.

With the second protocol, i.e. a dedicated CT protocol for "delayed contrast enhancement", a second imaging procedure is added to the CTCA protocol. Typically, this second procedure is performed 10-20 min after the first injection of contrast agent. The infarct zone will then be depicted as a hyperdense area, with increased uptake of the contrast agent, compared with the normal myocardium. This is because the contrast agent accumulates in the necrotic tissue (as also known from delayed enhancement MRI techniques) [46]. For optimal results of CT late enhancement imaging in myocardial infarctions, the applied
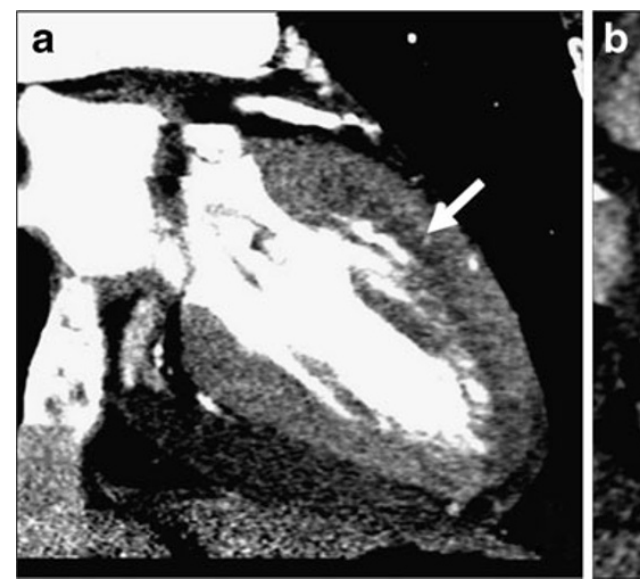

Fig. 4 Myocardial infarction and corresponding myocardial perfusion defect caused by a subtotal occlusion of a left anterior descending coronary artery (LAD) in a 57-year old female patient with multiple cardiovascular risk factors. a Longitudinal view of the left ventricle (multiplanar reformat) demonstrating a large perfusion defect in the anterior wall that is also depicted in the short-axis view (arrows, b). c As demonstrated in curved maximum intensity projection, the subtotal occlusion was located in the mid LAD segment just distal to a stent in the proximal coronary segment, which had been placed 3 months earlier due to a significant lesion detected on elective invasive angiography 
amount of contrast agent should be rather high. Mahnken et al. [47] have shown excellent results in animal studies, using $2 \mathrm{ml}$ per $\mathrm{kg}$ body weight or even more. However, this would increase the required amount of contrast agent in patients. For a patient of $75 \mathrm{~kg}$, a typical CT coronary angiogram would be performed with about $60-100 \mathrm{ml}$ of contrast agent (depending on the CT system generation used). For an optimised delayed enhancement acquisition, the optimal amount of contrast agent would be higher, e.g. within the range of $100-150 \mathrm{ml}$. In general, the late enhancement effect was primarily found in recent infarctions, but also reported to be detectable in chronic infarctions [47]. Habis et al. [48] could show perfect results by immediately injecting contrast agent via the coronary angiography catheter that was placed in the culprit coronary artery early after an acute myocardial infarction. Attempts to further optimise the CT late enhancement technique include usage of lower tube voltages [49], or reconstruction of thicker slices to be able to reduce radiation dose and still maintain a sufficiently high contrast-to-noise ratio. Even with these adaptations, so far, the detection of myocardial delayed enhancement still requires additional imaging procedures with an increased application of radiation dose to the patient and prolonged examination times, and it is therefore not being used extensively in clinical routine today. On the other hand, a comprehensive CT protocol including CTCA, display of myocardial perfusion defects and potentially myocardial viability and function would be a highly desirable application in CAD patients [50]. Therefore, it seems very probable that future studies on CT viability imaging will further enhance this technique and make it applicable in clinical routine, e.g. even using unenhanced CT techniques, as published recently [51].

\section{CT perfusion imaging}

The potential of CT to quantify myocardial perfusion using a "real" perfusion technique, i.e. a repeated, time-resolved, dynamic CT during myocardial wash-in and wash-out of the contrast agent, was already recognised in the 1980s and 1990s using electron beam computed tomography (EBCT). In animal models and healthy volunteers, early studies indicated the general ability of EBCT to determine blood flow and the effect of adenosine by using indicator dilution methods [52, 53]. Results from Lerman et al. [54] suggested that EBCT allowed the haemodynamic effect of a variety of vasoactive agents to be determined. More recently, Daghini et al. [55] and George et al. [56] showed similar results for 64-slice CT in an animal study.

Up to now, the technical challenges of time-resolved CT myocardial perfusion imaging have been substantial. A long acquisition time, i.e. a long temporal coverage of the myocardial contrast passage, has to be ensured, resulting in a mandatory imaging time of approximately 30-40 s. High temporal resolution is required for (semi-)quantification of perfusion data, and therefore, the interval between imaging at identical positions should not exceed one or two heartbeats. While with earlier CT generations (e.g. 64slice $\mathrm{CT}$ ) the anatomical coverage was limited to the detector width of a given CT system (typically around $4 \mathrm{~cm}$ coverage for 64-slice CT), and thus, only parts of the myocardium could be covered in a dynamic fashion, recent technical developments have changed this paradigm. Today, there are two technical options to cover the whole myocardium for dynamic perfusion imaging. First, CT with wide detectors, e.g. 256- or 320-slice CT, covering up to $16 \mathrm{~cm}$ without table movement [57], or second, the latest generation of dual-source CT systems, enabling a so-called "shuttle mode", which enables the composition of two adjacent slabs of myocardium of $4 \mathrm{~cm}$ each, resulting in nearly $8 \mathrm{~cm}$ of myocardial coverage in a dynamic fashion [58]. Overall, a perfusion sequence on MDCT is comparable to an ECG-triggered test bolus technique. To obtain a reasonable signal-time curve with a modest amount of contrast agent, high speed injections with a saline flush technique are preferable to acquire good quality signal-time curves (e.g. $\sim 40-50 \mathrm{ml}$ of contrast agent injected at a flow rate of about $5-8 \mathrm{ml} / \mathrm{s}$ ).

While all previous efforts at CT myocardial perfusion imaging were ROI-based, i.e. measuring the time-density curves in a small portion of the myocardium defined by a region-of-interest (ROI), a recent study extended these principles into a quantitative three-dimensional (3D) imaging technique, permitting regional myocardial blood flow (MBF) to be derived in patients in combination with CTbased morphological assessment of CAD, i.e. CTCA. Furthermore, using a second-generation dual-source CT, this study by Bamberg et al. [58] was the first to combine sufficient anatomical coverage, i.e. the whole myocardium, with a sufficiently high temporal resolution and stability of HU values, testing for stress-induced hypoperfusion in patients with known CAD (Fig. 5).

There are many technical aspects that need further optimisation in cardiac CT perfusion imaging. Even if modern techniques for reduction of radiation exposure are applied, the study protocol reported in the study by Bamberg et al. [58] still resulted in a comparably high total radiation exposure of an approximately 12-mSv-equivalent radiation dose (including the perfusion imaging and a prospective CTCA). On the other hand, this radiation exposure is similar to that of established retrospective CTCA examinations on 64-slice CT (with a reported median of $12 \mathrm{mSv}$ in a large multicentre study [59]) and comparable with or even lower than nuclear perfusion imaging, the clinical standard for the assessment of myocardial perfusion (radiation exposure for stress-only 

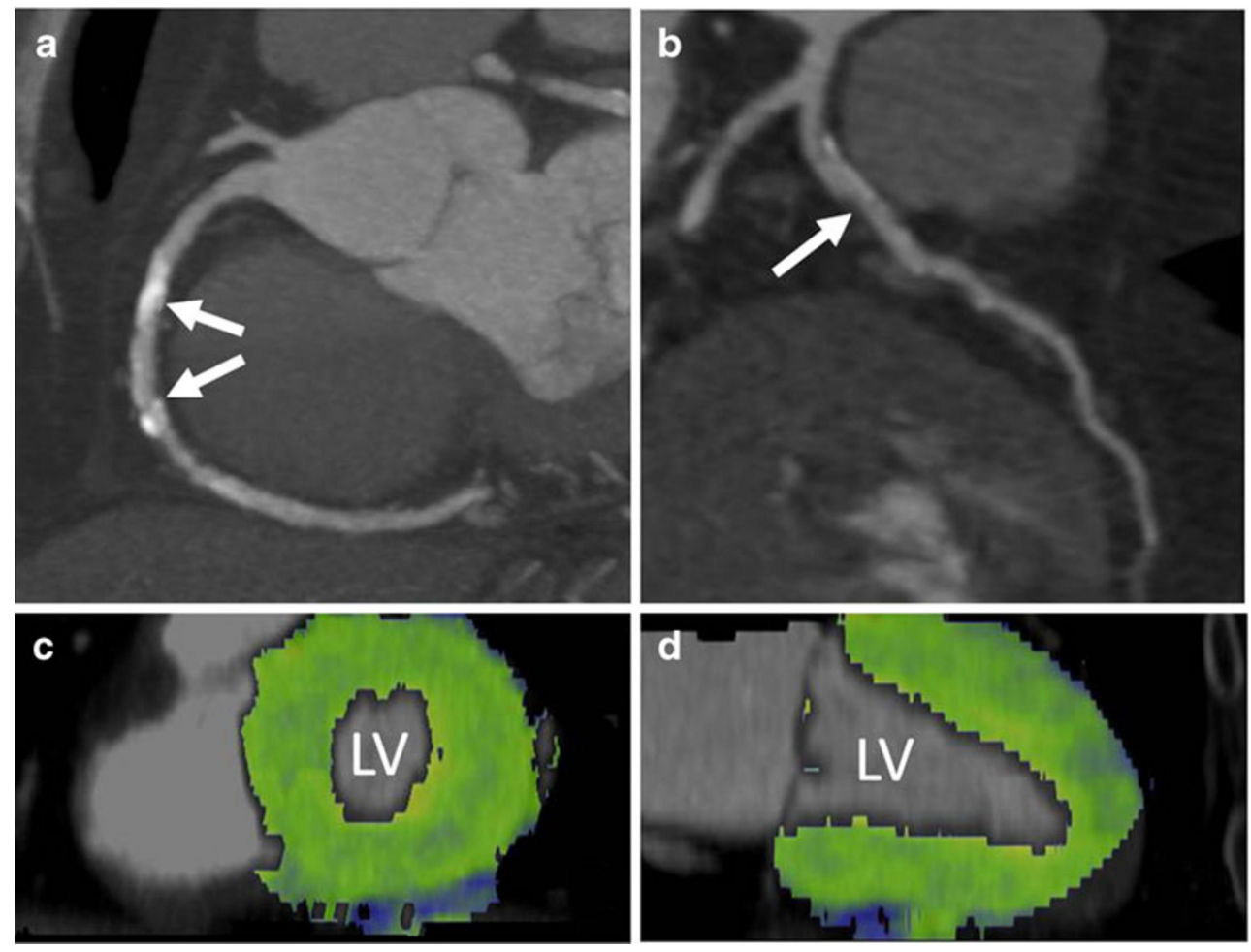

Fig. 5 A 71-year-old male patient who presented with stable chest pain syndrome and a history of cigarette smoking, hyperlipidaemia and hypertension. His right (a) and left anterior descending coronary arteries (b) demonstrate the presence of calcified and non-calcified plaque in all proximal vessel segments. The middle segment of the RCA has extensive calcification (arrows). Owing to limited evaluability of the coronary lumen, the presence of significant coronary

$99 \mathrm{~m}$ technetium (Tc) sestamibi is approximately $10.0 \mathrm{mSv}$, but that for 2-day ${ }^{99 \mathrm{~m}} \mathrm{Tc}$ sestamibi or ${ }^{201} \mathrm{Tl}$ is $20.0 \mathrm{mSv}$ or higher [60]). Further technical research will be necessary in CT myocardial perfusion imaging, to develop strategies to reduce radiation exposure and determine the impact of heart rate and different injection protocols on image quality, diagnostic accuracy and radiation exposure. Further validation studies in larger patient cohorts need to be carried out with standardised procedures for CT myocardial perfusion imaging, to prove the robustness and reproducibility of the technique. Comparisons using adequate gold standards (fractional flow reserve, MRI, or SPECT) are warranted to fully determine the diagnostic accuracy of cardiac CT for the quantification of myocardial perfusion defects in a blinded fashion. If confirmed, larger randomised diagnostic trials will be necessary to determine whether myocardial perfusion imaging by $\mathrm{CT}$ will be beneficial compared with established options using appropriate clinically relevant endpoints. Also, to prove the usefulness of a combined CT approach implementing CTCA with CT myocardial perfusion imaging, information on myocardial viability is of major importance, to differentiate between hypoperfused but viable from hypoperfused but necrotic myocardium. artery stenosis could not be ruled out. As part of a research protocol, the subject underwent dynamic adenosine-mediated stress myocardial perfusion imaging $(100 \mathrm{kV}, 175 \mathrm{mAs} / \mathrm{rot})$ over a period of $30 \mathrm{~s}$. Fusion images of the left ventricle (LV) in long axis (c) and short axis (d) demonstrate homogeneous myocardial blood flow without any evidence of stress-induced myocardial hypoperfusion. The measured myocardial blood flow was approximately $125 \mathrm{ml} / \mathrm{min} / 100 \mathrm{~g}$

Hence, a third CT acquisition for CT late enhancement would be mandatory in such a comprehensive CT approach. Also, the protocol includes the administration of adenosine, a short-lasting vasodilator, and continuous ECG monitoring. Contraindications for adenosine include Wolff-ParkinsonWhite (WPW) syndrome, atrioventricular (AV) block and sick sinus syndrome as well as asthma. Besides the development of dose reduction algorithms and optimised contrast agent injection protocols for dynamic myocardial perfusion imaging by $\mathrm{CT}$, work on dedicated reconstruction algorithms that optimise the contrast-to-noise ratio (CNR) might help further reduce radiation exposure.

\section{MRI for CAD: "the one-stop-shop"?}

\section{General considerations in cardiac MRI}

As emphasised earlier, CAD represents a relatively simple description of a disease that in fact consists of a complex array of different sub-entities, especially with regard to the extent, impact and effect upon the heart and its individual structures. MRI offers a wide variety of techniques that may 
be applied in patients with suspected or known CAD. The overall and final imaging strategy for the assessment of $\mathrm{CAD}$ and its sequelae, however, has to be chosen based on the clinical background information and the intended question for further therapeutic decisions. Although cardiac MRI has long been considered a possible "one-stop-shop" imaging technique in $\mathrm{CAD}$, this vision is rather theoretical than applicable in a clinical routine setting. Imaging strategies in cardiac MR could be addressed in various fashions; in the following overview, they will be highlighted according to technical issues as well as important clinical scenarios.

Assessment of coronary artery stenoses and myocardial ischaemia

\section{Coronary artery MRI}

Direct assessment of the coronary arteries and assessment of potential coronary artery stenoses are considered the holy grail of cardiac imaging. Although such an approach does only assess morphological changes, without any information on the haemodynamic relevance of a given stenoses, non-invasive MR coronary angiography has always been, and still remains, the ultimate goal for cardiac MRI. Ever since the first promising reports on coronary MR angiography in the early 1990s, with reported sensitivities and specificities of about $90 \%$ for $\geq 50 \%$ stenosis [61, 62], numerous technical approaches to coronary MR angiography have been proposed and implemented. Although software techniques as well as hardware have substantially changed, MR has not been able to either come to reproduce these promising early results, or to match results from the various generations of MDCT systems, except in cases with high coronary calcium score where CTCA may be of limited use [63]. Technical improvements incorporated various data acquisition strategies such as respiratory-gated coronary MR angiography, i.e. navigator techniques [64], as well as various strategies of whole heart coronary MR angiography with respiratory gating (Fig. 6) [65-68].

Clinical results though have generally not substantially improved with these technical developments, although overall imaging time has shortened. A major multi-centre study on 3D coronary MR angiography (on a single vendor platform) reported a sensitivity of $93 \%$ (in assessable segments) for any CAD $>50 \%$, while the specificity was as low as $42 \%$ [69]. Schuetz et al. [70] recently published a pooled sensitivity and specificity of coronary MR angiography of $87 \%$ and $70 \%$, respectively, for the diagnosis of a $\geq 50 \%$ coronary artery stenosis. These data are based on a meta-analysis incorporating 20 studies on coronary MRA (989 patients) that have been published from 1999 to 2009. The current superiority of MDCT coronary angiography has been demonstrated by a pooled sensitivity of $97 \%$ and specificity of $87 \%$, not even focusing on studies using only the most modern $\mathrm{CT}$ techniques and heart rate control [70].

Regarding the development of coronary MR angiography techniques, the following trends have consistently been reported:

- Three-dimensional steady-state free precession (SSFP) techniques allow for a higher image quality and CNR (blood/myocardium) than 3D gradient recalled echo (GRE) approaches [71, 72]

- The use of gadolinium-based contrast agents (GBCA) further improve CNR, irrespective of whether extracellular or intravascular compounds have been applied $[68,73,74]$

The use of higher field-strength (3 Tesla) and/or dedicated multi-element array coils for signal reception further pushed coronary MRA and resulted in improved signal-to-noise ratio (SNR) and CNR values [66, 73, 75]. The latter allows for either the improvement of spatial resolution or the reduction of imaging time with the use of
Fig. 6 Whole-heart coronary MRA (with slow Gd-chelate infusion) in a 24-year-old patient who had complained about recurrent chest pain on exertion since his childhood. The data set demonstrates a normal RCA (long arrow) and a myocardial bridging of the LAD with a minor bending along its course (short arrows)
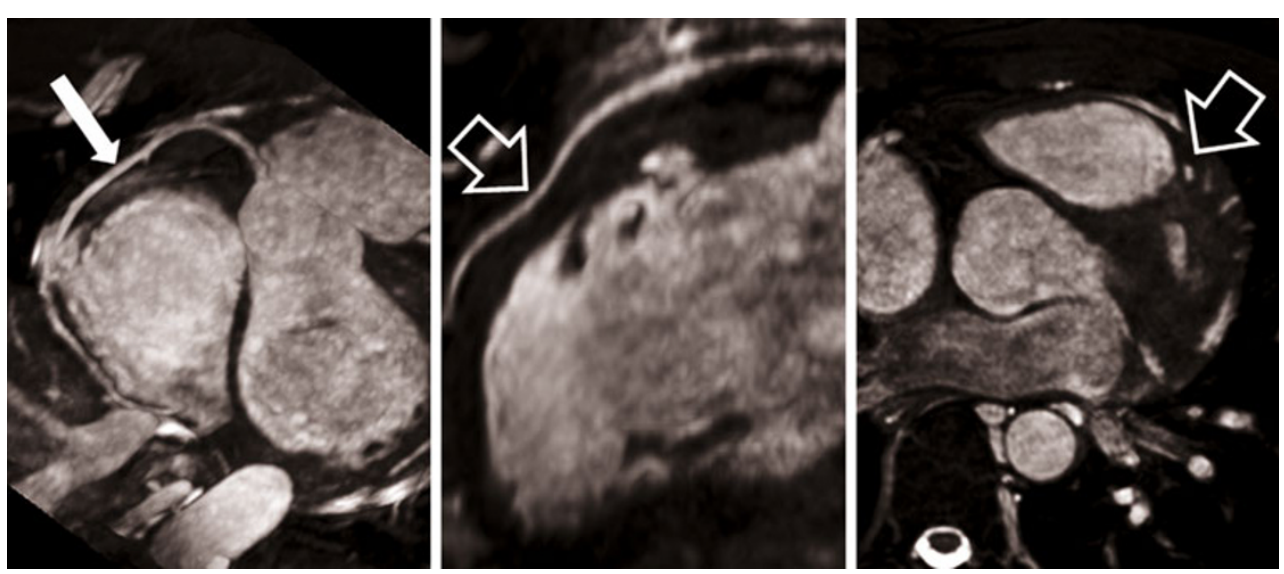
higher acceleration factors (e.g. applying parallel imaging techniques) [76]. Yang et al. [77] recently were able to demonstrate a sensitivity of $93 \%$ and a specificity of $89 \%$ for $>50 \%$ stenosis on a per-vessel basis, and $94 \%$ and $82 \%$, respectively, on a per-patient basis. Although not yet implemented for all coronary MR angiography studies, the use of pharmacological patient preparation for heart rate control (e.g. beta-blocker) or coronary vessel diameter (e.g. nitroglycerine) is increasingly being used and does show benefits in image quality [78, 79].

Taking into account all imaging preparation and scouting/ localising procedures, coronary MR angiography still remains a time-consuming and labour-intensive method. Although its useful application in a CAD population is still questionable, the technique may already today be routinely applied in the evaluation of proximal coronary artery anomalies, a possible cause of sudden coronary death in young athletes [80].

\section{Myocardial perfusion imaging}

Besides the direct visualisation of coronary artery stenosis, the indirect assessment of possible underlying stenotic CAD can also be rendered by MRI, using myocardial perfusion imaging. Rather than imaging of a coronary stenosis, this approach focuses on the assessment of a possible haemodynamic impact of a coronary artery lumen reduction on the downstream myocardial blood supply.

First-pass myocardial perfusion imaging is based on the use of gadolinium-based contrast agents (GBCA) as a tracer, and fast T1-weighted gradient recalled echo (GRE) or echoplanar imaging (EPI) hybrid techniques [81]. Rapid update imaging with a single-heart-beat resolution is required and allows for tracing of an extracellular contrast agent passing through the right ventricle (RV), the left ventricle (LV) and the myocardium. Regarding the assessment of the potential haemodynamic significance of a given coronary artery stenosis though, the use of proper stress testing is necessary in combination with myocardial perfusion imaging, as coronary luminal changes may compensate for a wide range of coronary luminal changes and therefore mask the presence of even high-grade lesions $[82,84]$. While dipyridamole and adenosine are at present most commonly used for vasodilation and thereby induce stress while performing MR myocardial perfusion imaging, newer vasodilators with more selective mechanisms may shortly be available that would ease the use in asthmatic patients or patients with chronic obstructive pulmonary disease (COPD) [83, 84].

The general principle of first-pass MR myocardial perfusion imaging has been evaluated by Wilke et al. [85, 86], as well as by other research groups, and has mainly been based on animal models that allow for comparison with reference standards (e.g. microspheres). Although the technique would in theory allow for a quantitative assessment of myocardial perfusion, the complex work-up of the MRI data and the lack of standardised postprocessing methods for perfusion quantification still prevent the technique from being used in a clinical workflow. Hence, most centres still rely on the visual interpretation of perfusion data sets (Fig. 7). In a recent meta-analysis, stress myocardial perfusion imaging has been reported with a
Fig. 7 Combined stress (upper row) and rest (lower row) first-pass myocardial perfusion imaging $(0.5 \mathrm{mmol} / \mathrm{kg} \mathrm{BW})$ demonstrating a reversible subendocardial perfusion deficit (arrows) in the inferioseptal and inferior aspect of the LV myocardium
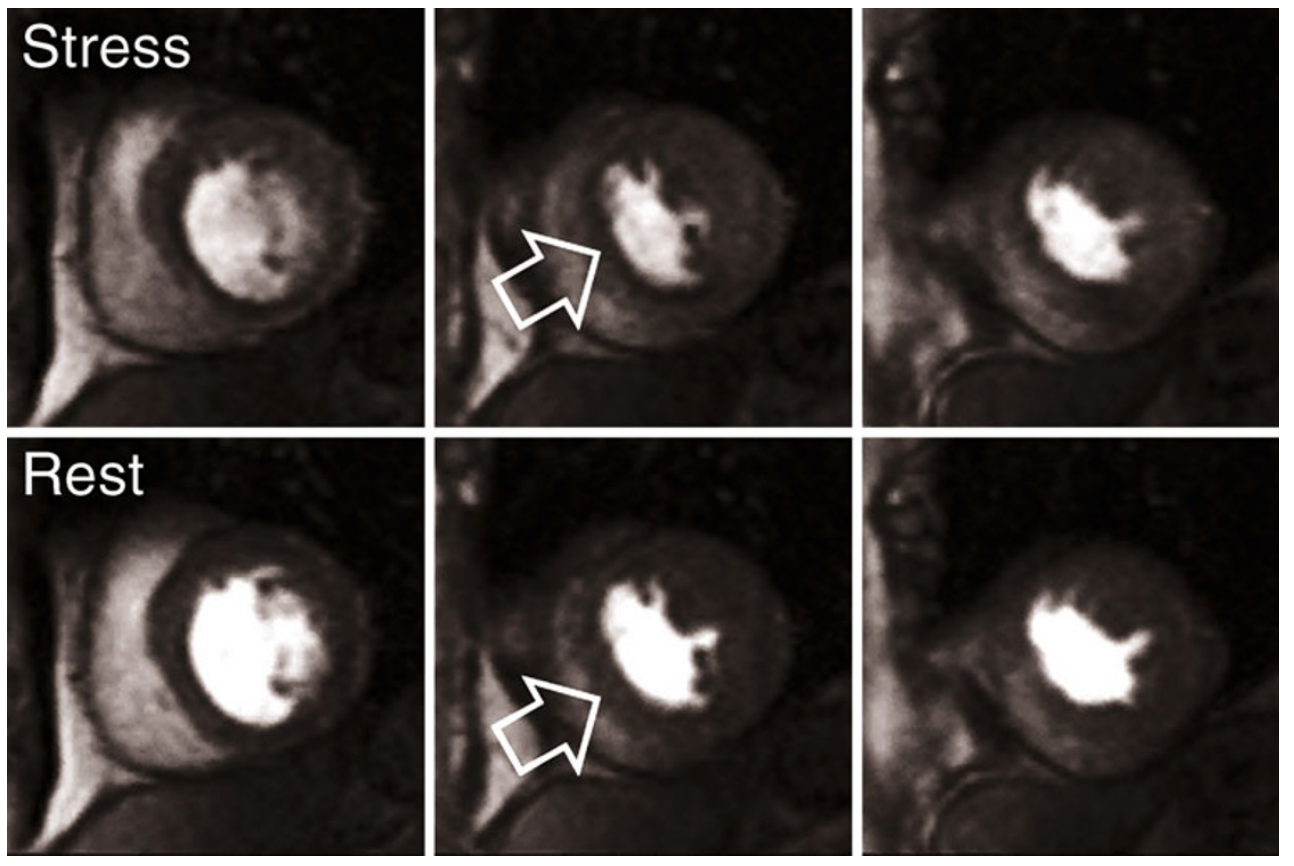
pooled sensitivity and specificity of $91 \%$ and $81 \%$, including studies with qualitative and quantitative approaches for the detection of a $\geq 50 \%$ diameter stenosis [87]. The benefit of quantitative analysis, though, has most recently been pointed out again by Patel et al. [88], and especially holds true for triple-vessel disease patients. Possible discrepancies of MR perfusion imaging with regard to standards such as quantitative coronary angiography (QCA) may not be related to the technical shortcomings of either imaging technique, but to the limitations of QCA in the assessment of the haemodynamic significance of a stenosis. While first-pass myocardial perfusion imaging evaluates functional aspects, QCA demonstrates morphological features, but delivers no functional information. Schwitter et al. [89] have demonstrated a higher sensitivity and specificity of first-pass perfusion imaging in comparison with PET definitions of ischaemia $(91 \%$ and $94 \%$, respectively) compared with the QCA diagnosis of $>50 \%$ coronary artery stenosis ( $87 \%$ and $85 \%$, respectively). The limited accuracy of QCA in the assessment of the haemodynamic severity of a given stenosis, especially in intermediate lesions, has been pointed out repeatedly [90, 91]. Regarding MR myocardial perfusion, most recent studies in fact focused on the use of invasive fractional flow reserve (FFR) measurements, serving as the standard of reference [92-94]. Using an FFR of $<0.75$ as a cut-off value for the differentiation of haemodynamic significant versus non-significant lesions, first-pass MR perfusion imaging showed a sensitivity and specificity of $91 \%$ and 94\% [93].

Technical MR developments, such as the advent of parallel imaging algorithms or the ongoing widespread use of higher magnetic field strength certainly has an impact on first-pass myocardial perfusion imaging $[95,96]$. Owing to the shortening of data acquisition intervals in the cardiac cycle using multiple element receiver-coils and parallel imaging algorithms, the frequency and severity of dark rim artefacts can be reduced, and as such, false-positive results may be potentially reduced [95]. The use of 3-Tesla field strength has not only been shown to provide benefits with regard to SNR, CNR and the amplitude of the SI-time curve but also, and more importantly, a potential impact on the accuracy of first-pass myocardial perfusion results [96-99]. Cheng et al. [98] reported an increase of $82 \%$ to $90 \%$ and $90 \%$ to $98 \%$ for sensitivity and specificity, respectively, when moving from 1.5 to 3 Tesla in an intraindividual comparative study of MR stress perfusion imaging for the detection of significant coronary artery lesions.

On the other hand, also at 3 Tesla, imaging parameters need to be well balanced with regard to spatial resolution, SNR and data acquisition time windows, to provide optimal results without interfering artefacts [100]. Also, the volume of injected contrast agent has to be considered and adapted; as for a visual assessment, a higher dose of contrast agent is typically recommended than for a quantitative assessment. The approach of a dual bolus setting though may allow both analyses in the same setting $[88,101]$.

New imaging approaches under investigation for the assessment of myocardial perfusion include techniques such as blood oxygen level-dependent (BOLD) techniques that potentially allow for detection of the effects of regional variations in myocardial blood flow based on the intrinsic contrast mechanism of desoxyhaemoglobin. Studies have demonstrated a good correlation with reference standards, and again, the use of a higher magnetic field strength (e.g. 3 Tesla) shows promise for further improvements in BOLD imaging [102-104].

\section{Stress cine MRI}

While MR perfusion imaging focuses on the direct detection of ischaemia at the myocardial blood supply level, stress cine imaging provides an indirect approach to visualising the potential impact of hypoperfusion and thus impaired myocardial oxygen supply on wall motion [105]. The underlying principle is not different from stress echocardiography and according to the guidelines of the major societies, high-dose protocols with infusion rates of $10,20,30 \mu \mathrm{g} / \mathrm{kg} / \mathrm{min}$ up to a maximal dose of $40 \mu \mathrm{g} / \mathrm{kg} / \mathrm{min}$ were adopted for dobutamine stress cine MRI (with the possible additional use of atropine). Other than vasodilators, used for stress perfusion MRI, stress cine MRI is based on the use of dobutamine, with a positive inotropic and chronotropic effect. This leads to a more "physiological stress" and allows for a better definition of peak stress levels compared with "stress" induced by vasodilators [105].

Typical MRI techniques for dobutamine stress imaging are based on standard functional techniques such as cine SSFP. The application of dobutamine stress in the MR environment though necessitates close patient monitoring with adequate, MR-compatible equipment. At different stress levels, cine MR images are being acquired and screened for newly developed wall motion abnormalities as a marker of insufficient myocardial oxygen supply. Nagel et al. [106] reported a sensitivity and specificity of $86 \%$ and $86 \%$ for diagnosis of $\geq 50 \%$ stenosis. The improved results compared with stress echocardiography in this study, though, were mainly based on the fact that MRI performs better than echocardiography in patients with poor acoustic windows. The results of this study are in good concordance with pooled data from a recent meta-analysis. Based on 14 studies applying stress cine MRI (mainly dobutamine use), Nandalur et al. [87] evaluated an overall sensitivity of $83 \%$ with a specificity of $86 \%$, for the detection of significant CAD. 


\section{Assessment of myocardial viability}

Total or temporary occlusion of a coronary artery may result in temporary ischaemic damage or myocardial death and necrosis in the downstream coronary artery territory. The extent of myocardial death in the setting of myocardial infarction has a substantial impact on the outcome of the patient. Myocardial infarction and its sequelae not only show a major impact on cardiac function and possible subsequent cardiac failure, but also on the risk of inducing severe arrhythmias.

MRI of myocardial viability has substantially changed over the last two decades. The evaluation of late gadolinium enhancement (LGE) techniques has pushed the envelope for the clinical routine assessment of myocardial viability using MR techniques. Before the use of LGE imaging assessment of myocardial viability using $\mathrm{MR}$ approaches has been based on low-dose $(5-10 \mu \mathrm{g} / \mathrm{kg} / \mathrm{min})$ dobutamine stress cine MRI [107, 108]. Early studies by Kim et al. [109, 110] evaluated the correlation of LGE of the myocardium and the presence of myocardial viability and necrosis in animal models. In early patient studies, the impact of this imaging technique on the prediction of patient outcome after myocardial infarction and revascularisation procedures has been demonstrated [111, 112]. LGE imaging offers the ability to assess the transmural extent of residual viability in patients after myocardial infarction. As a major pre-requisite in the use of LGE techniques in myocardial ischaemia, it has to be considered that the extent of myocardial infarction is typically related to coronary artery supply territories and that the development of necrosis starts at the subendocardial level propagating towards the epicardial border over time (Fig. 8) [113].
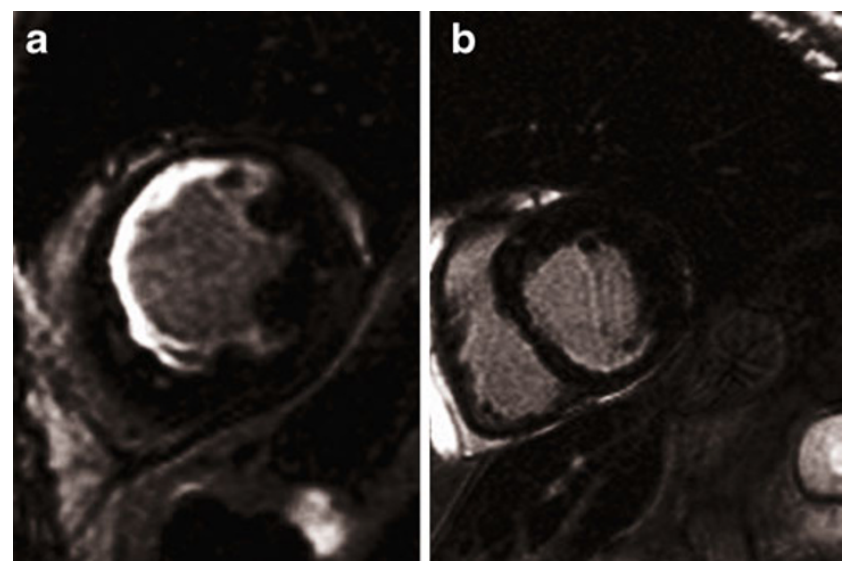

Fig. 8 Differentiation of transmural extent of myocardial infarction using late gadolinium enhancement (LGE) imaging. While the left image (a) demonstrates an almost transmural LGE consistent with only minor residual viable tissue, the patient on the right (b) only suffered from a smaller subendocardial myocardial infarction in the RCA territory
Sequence techniques for assessment of LGE are typically based on inversion recovery (IR) T1-weighted gradient recalled echo (GRE) techniques, with additional nulling [optimisation of inversion times (TI)] of the normal myocardium [114]. This approach allows for high differences in signal intensity of viable versus non-viable myocardium. With ongoing technical developments, changes in imaging approaches have been proposed and incorporated into clinical viability imaging. As segmented data acquisitions in MR rely on constant heart rates and patient cooperation with consistent breath-holding, image quality is substantially reduced in patients with severe arrhythmia or non-cooperative patients. Huber et al. [115] evaluated the use of single-shot LGE imaging techniques in combination with SSFP read-out in patients with myocardial infarctions and demonstrated excellent correlation of infarct size and volume compared with standard segmented IR techniques. In addition to the elimination of breathing artefacts, this technique can also be used for a substantial shortening of MRI time. As the image contrast of standard LGE imaging techniques relies on optimal TI settings, this approach is substantially operator-dependent. The use of advanced image calculation and processing techniques, as proposed by Kellman et al. [116], has led to the development of phase-sensitive IR techniques to alleviate the effects of suboptimal TI settings on overall image contrast. With regard to the volumetric accuracy of myocardial infarction this approach has been shown to demonstrate constant volumes compared with suboptimal TI settings with changing image contrast [117]. For LGE imaging, the use of higher magnetic field strength also demonstrates benefits with regard to signal levels. CNR levels (viable to non-viable myocardium) are typically higher at 3 Tesla although the overall image quality is not necessarily affected by this change [118, 119].

Regarding the differentiation of acute versus chronic infarction, the sole application of LGE imaging techniques is limited in its diagnostic use. The presence of LGE can be assessed as early as $1 \mathrm{~h}$ after infarction [120], but various mechanisms and principles maintain the effect of LGE in the infarct into its chronic stage [121]. Further differentiation can be provided by the additional use of T2-weighted oedema imaging. LGE imaging in the early stage of myocardial infarction not only allows the assessment of the extent of necrotic myocardium but also the possible delineation of areas of microvascular obstruction (MO), which, in addition to the overall infarct size, shows a correlation with the patient's outcome and degree of LV remodelling $[122,123]$. Although several studies evaluated the degree of MO in acute myocardial infarction using early and late gadolinium enhancement imaging, which technique has the better predictive value in terms of LV remodelling remains somewhat controversial [124-126]. 


\section{Conclusions}

MR and CT imaging are emerging as the most promising complementary imaging techniques in the primary diagnosis of CAD and for coronary atherosclerotic disease detection. For the detection or exclusion of significant CAD, both cardiac CT-including coronary calcium screening and non-invasive coronary angiography-and cardiac MR, applying stress function and stress perfusion imaging, are becoming widely available and are increasingly being applied in clinical routine. Owing to their high negative predictive value, especially in combining two or more of these applications, the presence of CAD can be excluded with a high probability, if patients are selected correctly. Today, invasive coronary catheterisation cannot fully be replaced by these techniques, and this should not be the primary goal of future investigations. The first goal might be a reduction of diagnostic invasive catheterisation without angioplastic intervention, by a combined and carefully selected approach using cardiac CT and/or MRI.

\section{References}

1. Gibbons RJ, Chatterjee K, Daley J et al (1999) ACC/AHA/ACPASIM guidelines for the management of patients with chronic stable angina: a report of the American College of Cardiology/ American Heart Association task force on practice guidelines (Committee on management of patients with chronic stable angina). J Am Coll Cardiol 33:2092-2197

2. Stein PD, Beemath A, Kayali F, Skaf E, Sanchez J, Olson RE (2006) Multidetector computed tomography for the diagnosis of coronary artery disease: a systematic review. Am J Med 119:203-216

3. Hendel RC, Patel MR, Kramer CM et al (2006) ACCF/ACR/ SCCT/SCMR/ASNC/NASCI/SCAI/SIR 2006 appropriateness criteria for cardiac computed tomography and cardiac magnetic resonance imaging: a report of the American College of Cardiology Foundation Quality Strategic Directions Committee Appropriateness Criteria Working Group, American College of Radiology, Society of Cardiovascular Computed Tomography, Society for Cardiovascular Magnetic Resonance, American Society of Nuclear Cardiology, North American Society for Cardiac Imaging, Society for Cardiovascular Angiography and Interventions, and Society of Interventional Radiology. J Am Coll Cardiol 48:1475-1497

4. Alkadhi H (2009) Radiation dose of cardiac CT-what is the evidence? Eur Radiol 19:1311-1315

5. Alkadhi H, Stolzmann P, Scheffel H et al (2008) Radiation dose of cardiac dual-source CT: the effect of tailoring the protocol to patient-specific parameters. Eur J Radiol 68:385-391

6. Shuman WP, Branch KR, May JM et al (2008) Prospective versus retrospective ECG gating for 64-detector CT of the coronary arteries: comparison of image quality and patient radiation dose. Radiology 248:431-437

7. Klass O, Jeltsch M, Feuerlein S et al (2009) Prospectively gated axial CT coronary angiography: preliminary experiences with a novel low-dose technique. Eur Radiol 19:829-836

8. Arnoldi E, Johnson TR, Rist C et al (2009) Adequate image quality with reduced radiation dose in prospectively triggered coronary CTA compared with retrospective techniques. Eur Radiol 19:2147-2155

9. Scheffel H, Alkadhi H, Leschka S et al (2008) Low-dose CT coronary angiography in the step-and-shoot mode: diagnostic performance. Heart 94:1132-1137

10. Ertel D, Lell MM, Harig F, Flohr T, Schmidt B, Kalender WA (2009) Cardiac spiral dual-source CT with high pitch: a feasibility study. Eur Radiol 19:2357-2362

11. Lell M, Hinkmann F, Anders K et al (2009) High-pitch electrocardiogram-triggered computed tomography of the chest: initial results. Invest Radiol 44:728-733

12. Lell M, Marwan M, Schepis T et al (2009) Prospectively ECGtriggered high-pitch spiral acquisition for coronary $\mathrm{CT}$ angiography using dual source $\mathrm{CT}$ : technique and initial experience. Eur Radiol 19:2576-2583

13. Sommer WH, Schenzle JC, Becker CR et al (2009) Saving dose in Triple-Rule-Out computed tomography examination using a high-pitch dual spiral technique. Invest Radiol 45:64-71

14. Leschka S, Stolzmann P, Desbiolles L et al (2009) Diagnostic accuracy of high-pitch dual-source CT for the assessment of coronary stenoses: first experience. Eur Radiol 19:2896-2890

15. Maurovich-Horvat P, Ferencik M, Bamberg F, Hoffmann U (2009) Methods of plaque quantification and characterization by cardiac computed tomography. J Cardiovasc Comput Tomogr 3 (Suppl 2):S91-S98

16. Pundziute G, Schuijf JD, Jukema JW et al (2007) Prognostic value of multislice computed tomography coronary angiography in patients with known or suspected coronary artery disease. $\mathrm{J}$ Am Coll Cardiol 49:62-70

17. van Werkhoven JM, Schuijf JD, Gaemperli O et al (2009) Prognostic value of multislice computed tomography and gated single-photon emission computed tomography in patients with suspected coronary artery disease. J Am Coll Cardiol 53:623-632

18. Leber AW, Becker A, Knez A et al (2006) Accuracy of 64-slice computed tomography to classify and quantify plaque volumes in the proximal coronary system: a comparative study using intravascular ultrasound. J Am Coll Cardiol 47:672-677

19. Saur SC, Alkadhi H, Stolzmann P et al (2010) Effect of reader experience on variability, evaluation time and accuracy of coronary plaque detection with computed tomography coronary angiography. Eur Radiol 20:1599-1606

20. Saur SC, Alkadhi H, Desbiolles L, Szekely G, Cattin PC (2009) ACCURATUM: improved calcium volume scoring using a mesh-based algorithm — a phantom study. Eur Radiol 19:591598

21. Saur SC, Cattin PC, Desbiolles L, Fuchs TJ, Szekely G, Alkadhi $\mathrm{H}$ (2009) Prediction rules for the detection of coronary artery plaques: evidence from cardiac CT. Invest Radiol 44:483-490

22. Becker CR, Nikolaou K, Muders M et al (2003) Ex vivo coronary atherosclerotic plaque characterization with multidetector-row CT. Eur Radiol 13:2094-2098

23. Nikolaou K, Becker CR, Muders M et al (2004) Multidetectorrow computed tomography and magnetic resonance imaging of atherosclerotic lesions in human ex vivo coronary arteries. Atherosclerosis 174:243-252

24. Cademartiri F, Mollet NR, Runza G et al (2005) Influence of intracoronary attenuation on coronary plaque measurements using multislice computed tomography: observations in an ex vivo model of coronary computed tomography angiography. Eur Radiol 15:1426-1431

25. Cademartiri F, La Grutta L, Runza G et al (2007) Influence of convolution filtering on coronary plaque attenuation values: observations in an ex vivo model of multislice computed tomography coronary angiography. Eur Radiol 17:1842-1849

26. Maurovich-Horvat P, Hoffmann U, Vorpahl M, Nakano M, Virmani R, Alkadhi H (2010) The napkin-ring sign: CT signature 
of high-risk coronary plaques? JACC Cardiovasc Imaging 3:440-444

27. Hyafil F, Cornily JC, Feig JE et al (2007) Noninvasive detection of macrophages using a nanoparticulate contrast agent for computed tomography. Nat Med 13:636-641

28. Hendel RC, Budoff MJ, Cardella JF et al (2009) ACC/AHA/ ACR/ASE/ASNC/HRS/NASCI/RSNA/SAIP/SCAI/SCCT/ SCMR/SIR 2008 key data elements and definitions for cardiac imaging: a report of the American College of Cardiology/ American Heart Association Task Force on Clinical Data Standards (Writing Committee to Develop Clinical Data Standards for Cardiac Imaging). Circulation 119:154-186

29. Chae SC, Heo J, Iskandrian AS, Wasserleben V, Cave V (1993) Identification of extensive coronary artery disease in women by exercise single-photon emission computed tomographic (SPECT) thallium imaging. J Am Coll Cardiol 21: 1305-1311

30. Budoff MJ, Achenbach S, Blumenthal RS et al (2006) Assessment of coronary artery disease by cardiac computed tomography: a scientific statement from the American heart association committee on cardiovascular imaging and intervention, council on cardiovascular radiology and intervention, and committee on cardiac imaging, council on clinical cardiology. Circulation 114:1761-1791

31. Naito H, Saito H, Takamiya M et al (1992) Quantitative assessment of myocardial enhancement with iodinated contrast medium in patients with ischemic heart disease by using ultrafast x-ray computed tomography. Invest Radiol 27:436-442

32. Koyama Y, Mochizuki T, Higaki J (2004) Computed tomography assessment of myocardial perfusion, viability, and function. J Magn Reson Imaging 19:800-815

33. Higgins CB, Siemers PT, Newell JD, Schmidt W (1980) Role of iodinated contrast material in the evaluation of myocardial infarction by computerized transmission tomography. Invest Radiol 15:S176-S182

34. Higgins CB, Sovak M, Schmidt W, Siemers PT (1979) Differential accumulation of radiopaque contrast material in acute myocardial infarction. Am J Cardiol 43:47-51

35. Nagel E, Klein C, Paetsch I et al (2003) Magnetic resonance perfusion measurements for the noninvasive detection of coronary artery disease. Circulation 108:432-437

36. Mahnken AH, Bruners P, Katoh M, Wildberger JE, Gunther RW, Buecker A (2006) Dynamic multi-section CT imaging in acute myocardial infarction: preliminary animal experience. Eur Radiol $16: 746-752$

37. Nikolaou K, Sanz J, Poon M et al (2005) Assessment of myocardial perfusion and viability from routine contrastenhanced 16-detector-row computed tomography of the heart: preliminary results. Eur Radiol 15:864-871

38. Huber DJ, Lapray JF, Hessel SJ (1981) In vivo evaluation of experimental myocardial infarcts by ungated computed tomography. AJR Am J Roentgenol 136:469-473

39. Slutsky RA, Mattrey RF, Long SA, Higgins CB (1983) In vivo estimation of myocardial infarct size and left ventricular function by prospectively gated computerized transmission tomography. Circulation 67:759-765

40. Slutsky RA, Peck WW, Mancini GB, Mattrey RF, Higgins CB (1984) Myocardial infarct size determined by computed transmission tomography in canine infarcts of various ages and in the presence of coronary reperfusion. J Am Coll Cardiol 3:138-142

41. Schmermund A, Gerber T, Behrenbeck T et al (1998) Measurement of myocardial infarct size by electron beam computed tomography: a comparison with $99 \mathrm{mTc}$ sestamibi. Invest Radiol $33: 313-321$
42. Georgiou D, Bleiweis M, Brundage BH (1992) Conventional and ultrafast computed tomography in the detection of viable versus infarcted myocardium. Am J Card Imaging 6:228-236

43. Georgiou D, Wolfkiel C, Brundage BH (1994) Ultrafast computed tomography for the physiological evaluation of myocardial perfusion. Am J Card Imaging 8:151-158

44. Nieman K, van Ooijen P, Rensing B, Oudkerk M, de Feyter PJ (2001) Four-dimensional cardiac imaging with multislice computed tomography. Circulation 103:E62

45. Hilfiker PR, Weishaupt D, Marincek B (2001) Multislice spiral computed tomography of subacute myocardial infarction. Circulation 104:1083

46. Mahrholdt H, Wagner A, Holly TA et al (2002) Reproducibility of chronic infarct size measurement by contrast-enhanced magnetic resonance imaging. Circulation 106:2322-2327

47. Mahnken AH, Bruners P, Kinzel S et al (2007) Late-phase MSCT in the different stages of myocardial infarction: animal experiments. Eur Radiol 17:2310-2317

48. Habis M, Daoud B, Roger VL et al (2007) Comparison of 64-slice computed tomography planimetry and Doppler echocardiography in the assessment of aortic valve stenosis. J Heart Valve Dis 16:216-224

49. Mahnken AH, Bruners P, Muhlenbruch G et al (2007) Low tube voltage improves computed tomography imaging of delayed myocardial contrast enhancement in an experimental acute myocardial infarction model. Invest Radiol 42:123-129

50. Cury RC, Nieman K, Shapiro MD et al (2008) Comprehensive assessment of myocardial perfusion defects, regional wall motion, and left ventricular function by using 64-section multidetector CT. Radiology 248:466-475

51. Mahnken AH, Bruners P, Bornikoel CM, Kramer N, Guenther RW (2009) Assessment of myocardial edema by computed tomography in myocardial infarction. JACC Cardiovasc Imaging 2:1167-1174

52. Bell MR, Lerman LO, Rumberger JA (1999) Validation of minimally invasive measurement of myocardial perfusion using electron beam computed tomography and application in human volunteers. Heart 81:628-635

53. Gould RG, Lipton MJ, McNamara MT, Sievers RE, Koshold S, Higgins CB (1988) Measurement of regional myocardial blood flow in dogs by ultrafast CT. Invest Radiol 23:348-353

54. Lerman LO, Siripornpitak S, Maffei NL, Sheedy PF 2nd, Ritman EL (1999) Measurement of in vivo myocardial microcirculatory function with electron beam CT. J Comput Assist Tomogr 23:390-398

55. Daghini E, Primak AN, Chade AR et al (2007) Evaluation of porcine myocardial microvascular permeability and fractional vascular volume using 64-slice helical computed tomography (CT). Invest Radiol 42:274-282

56. George RT, Jerosch-Herold M, Silva C et al (2007) Quantification of myocardial perfusion using dynamic 64-detector computed tomography. Invest Radiol 42:815-822

57. Hsiao EM, Rybicki FJ, Steigner M (2010) CT coronary angiography: 256-slice and 320-detector row scanners. Curr Cardiol Rep 12:68-75

58. Bamberg F, Klotz E, Flohr T et al (2010) Dynamic myocardial stress perfusion imaging using fast dual-source CT with alternating table positions: initial experience. Eur Radiol 20:5

59. Hausleiter J, Meyer T, Hermann F et al (2009) Estimated radiation dose associated with cardiac CT angiography. JAMA 301:500-507

60. Einstein AJ, Moser KW, Thompson RC, Cerqueira MD, Henzlova MJ (2007) Radiation dose to patients from cardiac diagnostic imaging. Circulation 116:1290-1305 
61. Edelman RR, Manning WJ, Burstein D, Paulin S (1991) Coronary arteries: breath-hold MR angiography. Radiology 181:641-643

62. Manning WJ, Li W, Edelman RR (1993) A preliminary report comparing magnetic resonance coronary angiography with conventional angiography. N Engl J Med 328:828-832

63. Liu X, Zhao X, Huang J et al (2007) Comparison of 3D freebreathing coronary MR angiography and 64-MDCT angiography for detection of coronary stenosis in patients with high calcium scores. AJR Am J Roentgenol 189:1326-1332

64. Nikolaou K, Huber A, Knez A, Scheidler J, Petsch R, Reiser M (2001) Navigator echo-based respiratory gating for 3D-MR coronary angiography: Reduction of scan time using a sliceinterpolation technique. J Comput Assist Tomogr 25:378-387

65. Bi X, Deshpande V, Carr J, Li D (2006) Coronary artery magnetic resonance angiography (MRA): a comparison between the whole-heart and volume-targeted methods using a T2prepared SSFP sequence. J Cardiovasc Magn Reson 8:703-707

66. Bi X, Deshpande V, Simonetti O, Laub G, Li D (2005) Three-dimensional breathhold SSFP coronary MRA: a comparison between $1.5 \mathrm{~T}$ and 3.0 T. J Magn Reson Imaging 22: 206-212

67. Lai P, Bi X, Jerecic R, Li D (2009) A respiratory self-gating technique with 3D-translation compensation for free-breathing whole-heart coronary MRA. Magn Reson Med 62:731-738

68. Tang L, Merkle N, Schar M et al (2009) Volume-targeted and whole-heart coronary magnetic resonance angiography using an intravascular contrast agent. J Magn Reson Imaging 30: 1191-1196

69. Kim WY, Danias PG, Stuber M et al (2001) Coronary magnetic resonance angiography for the detection of coronary stenoses. $\mathrm{N}$ Engl J Med 345:1863-1869

70. Schuetz GM, Zacharopoulou NM, Schlattmann P, Dewey M (2010) Meta-analysis: noninvasive coronary angiography using computed tomography versus magnetic resonance imaging. Ann Intern Med 152:167-177

71. Spuentrup E, Buecker A, Stuber M et al (2003) Navigator-gated coronary magnetic resonance angiography using steady-statefree-precession: comparison to standard T2-prepared gradientecho and spiral imaging. Invest Radiol 38:263-268

72. Weber OM, Pujadas S, Martin AJ, Higgins CB (2004) Freebreathing, three-dimensional coronary artery magnetic resonance angiography: comparison of sequences. J Magn Reson Imaging 20:395-402

73. Bi X, Carr JC, Li D (2007) Whole-heart coronary magnetic resonance angiography at 3 Tesla in 5 minutes with slow infusion of Gd-BOPTA, a high-relaxivity clinical contrast agent. Magn Reson Med 58:1-7

74. Prompona M, Cyran C, Nikolaou K, Bauner K, Reiser M, Huber A (2009) Contrast-enhanced whole-heart MR coronary angiography at $3.0 \mathrm{~T}$ using the intravascular contrast agent gadofosveset. Invest Radiol 44:369-374

75. Bhat H, Yang Q, Zuehlsdorff S, Li K, Li D (2010) Contrastenhanced whole-heart coronary magnetic resonance angiography at $3 \mathrm{~T}$ using interleaved echo planar imaging. Invest Radiol 45:458-464

76. Yu J, Schar M, Vonken EJ, Kelle S, Stuber M (2009) Improved SNR efficiency in gradient echo coronary MRA with high temporal resolution using parallel imaging. Magn Reson Med 62:1211-1220

77. Yang Q, Li K, Liu X et al (2009) Contrast-enhanced whole-heart coronary magnetic resonance angiography at 3.0-T: a comparative study with X-ray angiography in a single center. J Am Coll Cardiol 54:69-76
78. Jin H, Zeng MS, Ge MY et al (2010) Influence of applying nitroglycerin in whole-heart free-breathing 3D coronary MR angiography. AJR Am J Roentgenol 194:927-932

79. Terashima M, Meyer CH, Keeffe BG et al (2005) Noninvasive assessment of coronary vasodilation using magnetic resonance angiography. J Am Coll Cardiol 45:104-110

80. Prakken NH, Cramer MJ, Olimulder MA, Agostoni P, Mali WP, Velthuis BK (2010) Screening for proximal coronary artery anomalies with 3-dimensional MR coronary angiography. Int $\mathrm{J}$ Cardiovasc Imaging 26:701-710

81. Hunold P, Schlosser T, Barkhausen J (2006) Magnetic resonance cardiac perfusion imaging - a clinical perspective. Eur Radiol 16:1779-1788

82. Wilson RF (1996) Assessing the severity of coronary-artery stenoses. N Engl J Med 334:1735-1737

83. Mekkaoui C, Jadbabaie F, Dione DP et al (2009) Effects of adenosine and a selective $\mathrm{A} 2 \mathrm{~A}$ adenosine receptor agonist on hemodynamic and thallium-201 and technetium-99 m-sestaMIBI biodistribution and kinetics. JACC Cardiovasc Imaging 2: $1198-1208$

84. Kwon DH, Cerqueira MD, Young R et al (2010) Lessons from regadenoson and low-level treadmill/regadenoson myocardial perfusion imaging: Initial clinical experience in 1263 patients. J Nucl Cardiol 17:853-857

85. Wilke N, Jerosch-Herold M, Wang Y et al (1997) Myocardial perfusion reserve: assessment with multisection, quantitative, first-pass MR imaging. Radiology 204:373-384

86. Wilke N, Simm C, Zhang J et al (1993) Contrast-enhanced first pass myocardial perfusion imaging: correlation between myocardial blood flow in dogs at rest and during hyperemia. Magn Reson Med 29:485-497

87. Nandalur KR, Dwamena BA, Choudhri AF, Nandalur MR, Carlos RC (2007) Diagnostic performance of stress cardiac magnetic resonance imaging in the detection of coronary artery disease: a meta-analysis. J Am Coll Cardiol 50:13431353

88. Patel AR, Antkowiak PF, Nandalur KR et al (2010) Assessment of advanced coronary artery disease: advantages of quantitative cardiac magnetic resonance perfusion analysis. J Am Coll Cardiol 56:561-569

89. Schwitter J, Nanz D, Kneifel S et al (2001) Assessment of myocardial perfusion in coronary artery disease by magnetic resonance: a comparison with positron emission tomography and coronary angiography. Circulation 103:2230-2235

90. Tobis J, Azarbal B, Slavin L (2007) Assessment of intermediate severity coronary lesions in the catheterization laboratory. J Am Coll Cardiol 49:839-848

91. Yong AS, Ng AC, Brieger D, Lowe HC, Ng MK, Kritharides L (2010) Three-dimensional and two-dimensional quantitative coronary angiography, and their prediction of reduced fractional flow reserve. Eur Heart J. doi:10.1093/eurheartj/ehq259

92. Rieber J, Huber A, Erhard I et al (2006) Cardiac magnetic resonance perfusion imaging for the functional assessment of coronary artery disease: a comparison with coronary angiography and fractional flow reserve. Eur Heart J 27:1465-1471

93. Watkins S, McGeoch R, Lyne J et al (2009) Validation of magnetic resonance myocardial perfusion imaging with fractional flow reserve for the detection of significant coronary heart disease. Circulation 120:2207-2213

94. Costa MA, Shoemaker S, Futamatsu H et al (2007) Quantitative magnetic resonance perfusion imaging detects anatomic and physiologic coronary artery disease as measured by coronary angiography and fractional flow reserve. J Am Coll Cardiol $50: 514-522$ 
95. Theisen D, Wintersperger BJ, Huber A, Dietrich O, Reiser MF, Schonberg SO (2007) Myocardial first pass perfusion imaging with gadobutrol: impact of parallel imaging algorithms on image quality and signal behavior. Invest Radiol 42:522-528

96. Theisen D, Wintersperger BJ, Huber A, Dietrich O, Reiser MF, Schonberg SO (2007) Myocardial perfusion imaging with gadobutrol: a comparison between 3 and 1.5 tesla with an identical sequence design. Invest Radiol 42:499-506

97. Su MY, Yang KC, Wu CC et al (2007) First-pass myocardial perfusion cardiovascular magnetic resonance at 3 Tesla. J Cardiovasc Magn Reson 9:633-644

98. Cheng AS, Pegg TJ, Karamitsos TD et al (2007) Cardiovascular magnetic resonance perfusion imaging at 3-tesla for the detection of coronary artery disease: a comparison with 1.5-tesla. J Am Coll Cardiol 49:2440-2449

99. Klumpp BD, Seeger A, Doesch C et al (2010) High resolution myocardial magnetic resonance stress perfusion imaging at $3 \mathrm{~T}$ using a $1 \mathrm{M}$ contrast agent. Eur Radiol 20:533-541

100. Di Bella EV, Parker DL, Sinusas AJ (2005) On the dark rim artifact in dynamic contrast-enhanced MRI myocardial perfusion studies. Magn Reson Med 54:1295-1299

101. Schwitter J, Wacker CM, van Rossum AC et al (2008) MRIMPACT: comparison of perfusion-cardiac magnetic resonance with single-photon emission computed tomography for the detection of coronary artery disease in a multicentre, multivendor, randomized trial. Eur Heart J 29:480-489

102. McCommis KS, Goldstein TA, Abendschein DR et al (2010) Roles of myocardial blood volume and flow in coronary artery disease: an experimental MRI study at rest and during hyperemia. Eur Radiol 20:2005-2012

103. McCommis KS, Zhang H, Goldstein TA et al (2009) Myocardial blood volume is associated with myocardial oxygen consumption: an experimental study with cardiac magnetic resonance in a canine model. JACC Cardiovasc Imaging 2:1313-1320

104. Dharmakumar R, Arumana JM, Tang R, Harris K, Zhang Z, Li D (2008) Assessment of regional myocardial oxygenation changes in the presence of coronary artery stenosis with balanced SSFP imaging at 3.0 T: theory and experimental evaluation in canines. J Magn Reson Imaging 27:1037-1045

105. Strach K, Meyer C, Schild H, Sommer T (2006) Cardiac stress MR imaging with dobutamine. Eur Radiol 16:2728-2738

106. Nagel E, Lehmkuhl HB, Bocksch W et al (1999) Noninvasive diagnosis of ischemia-induced wall motion abnormalities with the use of high-dose dobutamine stress MRI: comparison with dobutamine stress echocardiography. Circulation 99:763-770

107. Barilla F, De Vincentis G, Mangieri E et al (1999) Recovery of contractility of viable myocardium during inotropic stimulation is not dependent on an increase of myocardial blood flow in the absence of collateral filling. J Am Coll Cardiol 33:697-704

108. Sandstede JJ, Bertsch G, Beer M et al (1999) Detection of myocardial viability by low-dose dobutamine Cine MR imaging. Magn Reson Imaging 17:1437-1443

109. Kim RJ, Chen EL, Lima JA, Judd RM (1996) Myocardial GdDTPA kinetics determine MRI contrast enhancement and reflect the extent and severity of myocardial injury after acute reperfused infarction. Circulation 94:3318-3326

110. Kim RJ, Fieno DS, Parrish TB et al (1999) Relationship of MRI delayed contrast enhancement to irreversible injury, infarct age, and contractile function. Circulation 100:1992-2002
111. Kim RJ, Wu E, Rafael A et al (2000) The use of contrastenhanced magnetic resonance imaging to identify reversible myocardial dysfunction. N Engl J Med 343:1445-1453

112. Schvartzman PR, Srichai MB, Grimm RA et al (2003) Nonstress delayed-enhancement magnetic resonance imaging of the myocardium predicts improvement of function after revascularization for chronic ischemic heart disease with left ventricular dysfunction. Am Heart J 146:535-541

113. Kloner RA, Jennings RB (2001) Consequences of brief ischemia: stunning, preconditioning, and their clinical implications: part 1. Circulation 104:2981-2989

114. Simonetti OP, Kim RJ, Fieno DS et al (2001) An improved MR imaging technique for the visualization of myocardial infarction. Radiology 218:215-223

115. Huber A, Schoenberg SO, Spannagl B et al (2006) Single-shot inversion recovery TrueFISP for assessment of myocardial infarction. AJR Am J Roentgenol 186:627-633

116. Kellman P, Arai AE, McVeigh ER, Aletras AH (2002) Phasesensitive inversion recovery for detecting myocardial infarction using gadolinium-delayed hyperenhancement. Magn Reson Med 47:372-383

117. Huber AM, Schoenberg SO, Hayes C et al (2005) Phasesensitive inversion-recovery MR imaging in the detection of myocardial infarction. Radiology 237:854-860

118. Bauner KU, Muehling O, Wintersperger BJ, Winnik E, Reiser MF, Huber A (2007) Inversion recovery single-shot TurboFLASH for assessment of myocardial infarction at 3 Tesla. Invest Radiol 42:361-371

119. Klumpp BD, Sandstede J, Lodemann KP et al (2009) Intraindividual comparison of myocardial delayed enhancement MR imaging using gadobenate dimeglumine at $1.5 \mathrm{~T}$ and $3 \mathrm{~T}$. Eur Radiol 19:1124-1131

120. Schulz-Menger J, Gross M, Messroghli D, Uhlich F, Dietz R, Friedrich MG (2003) Cardiovascular magnetic resonance of acute myocardial infarction at a very early stage. J Am Coll Cardiol 42:513-518

121. Mahrholdt H, Wagner A, Judd RM, Sechtem U (2002) Assessment of myocardial viability by cardiovascular magnetic resonance imaging. Eur Heart J 23:602-619

122. Gerber BL, Rochitte CE, Melin JA et al (2000) Microvascular obstruction and left ventricular remodeling early after acute myocardial infarction. Circulation 101:2734-2741

123. Bekkers SC, Smulders MW, Passos VL et al (2010) Clinical implications of microvascular obstruction and intramyocardial haemorrhage in acute myocardial infarction using cardiovascular magnetic resonance imaging. Eur Radiol 20:2572-2578

124. Larose E, Rodes-Cabau J, Pibarot P et al (2010) Predicting late myocardial recovery and outcomes in the early hours of STsegment elevation myocardial infarction traditional measures compared with microvascular obstruction, salvaged myocardium, and necrosis characteristics by cardiovascular magnetic resonance. J Am Coll Cardiol 55:2459-2469

125. Cochet A, Lalande A, Lorgis L et al (2010) Prognostic value of microvascular damage determined by cardiac magnetic resonance in non ST-segment elevation myocardial infarction: comparison between first-pass and late gadolinium-enhanced images. Invest Radiol 45(11):725-732

126. de Waha S, Desch S, Eitel I et al (2010) Impact of early vs. late microvascular obstruction assessed by magnetic resonance imaging on long-term outcome after ST-elevation myocardial infarction: a comparison with traditional prognostic markers. Eur Heart J. doi:10.1093/eurheartj/ehq247 